\title{
On a cubic curve connected with the triangle.
}

\section{By Professor Allardice.}

In a recent number of the Annals of Mathematics* I have shown that the asymptotes of a conic circumscribed about a triangle are isotomic lines with reference to the triangle. By means of this theorem it is easy to find the locus of the centre of a conic, circumscribed about a given triangle, when one of the asymptotes passes through a fixed point.

If $(l, m, n)$ is the fixed point, we may write the equations of the asymptotes in the form

$$
\begin{aligned}
& \text { (1) } p a+q \beta+r \gamma=0 \\
& \text { (2) } a^{2} a / p+b^{2} \beta / q+c^{2} \gamma / r=0 \text {; subject to the condition } \\
& \text { (3) } p l+q m+r n=0
\end{aligned}
$$

Solving equations (1) and (3) for $p, q, r$, and substituting in (2), we get the result

$$
a^{2} a /(m \gamma-n \beta)+b^{2} \beta /(n a-l \gamma)+c^{2} \gamma /(l \beta-m a)=0 ;
$$

or, putting $a a=x, b \beta=y, c \gamma=z ; l a=\lambda, m b=\mu, n c=v$,

$$
x(\nu x-\lambda z)(\lambda y-\mu x)+y(\lambda y-\mu x)(\mu z-\nu y)+z(\mu z-\nu y)(\nu x-\lambda z)=0 .
$$

The equation may also be written in the forms

$$
\begin{aligned}
& \lambda \mu z\left(x^{2}+y^{2}-z^{2}\right)+\mu \nu x\left(y^{\prime \prime}+z^{2}-x^{2}\right)+v \lambda y\left(z^{2}+x^{2}-y^{\prime \prime}\right)-\left(\lambda^{2}+\mu^{2}+v^{2}\right) x y z=0 \\
&(x+y+z)\{\lambda \mu z(x+y-z)+\mu \nu x(y+z-x)+\nu \lambda y(z+x-y)\} \\
&-(\lambda+\mu+\nu)^{\prime \prime} x y z=0 .
\end{aligned}
$$

The curve is a cubic, with a double point at $(l, m, n)$; it meets the sides at their middle points, at the points where the lines joining $(l, m, n)$ to the vertices meet the sides, and at the points at infinity on the sides.

\footnotetext{
* Annals of Mathematics, Second Series, Vol. 2, No. 3.
} 
The asymptote parallel to $z$ is

$$
(\lambda+\mu+\nu)^{2} z=2 \nu(\lambda+\mu)(x+y+z)
$$

and the cubic may be written in the form

$$
\begin{aligned}
& \left\{2 \nu(\lambda+\mu)(x+y+z)-(\lambda+\mu+\nu)^{2} z\right\}\{\mu \nu x(y+z-x)+\nu \lambda y(z+x-y) \\
& +\lambda \mu z(x+y-z)\}+(\lambda+\mu+\nu)^{2} z(x+y-z)(\lambda \mu z-\mu \nu x-\lambda \nu y)=0 .
\end{aligned}
$$

This asymptote is inflexional if $\lambda=\mu$, that is, if $(l, m, n)$ lies on the median through the vertex opposite $z$.

The asymptotes meet in a point, if $(l, m, n)$ be at infinity (in which case the cubic breaks up into a parabola and the straight line at infinity); also if $(l, m, n)$ lie on the maximum inscribed ellipse.

It is convenient to refer the curve to the triangle, the vertices of which are the middle points of the sides of the original triangle. The equation then becomes

$$
\lambda^{2} y z(y+z)+\mu^{2} z x(z+x)+\nu^{2} x y(x+y)-2(\lambda \mu+\mu \nu+\nu \lambda) x y z=0,
$$

where $(\lambda, \mu, v)$ are now the coordinates of the given point (in the form $a l, b m, c n)$ with respect to the new triangle of reference.

The asymptotes are

$$
(\lambda+\mu+v)^{2}(x+y)=(\lambda+\mu)(\lambda+\mu+2 v)(x+y+z), \text { etc. ; }
$$

and the tangents at the double point are

$$
\mu \nu(\mu+v) x^{2}+v \lambda(\nu+\lambda) y^{2}+\lambda \mu(\lambda+\mu) z^{2}-2 \lambda \mu \nu(x y+y z+z x)=0 .
$$

The points of intersection of the asymptotes with the curve lie on the straight line

$$
\begin{aligned}
\mu^{2} v^{2}(\mu+\nu)(\mu+\nu+2 \lambda) x+\nu^{2} \lambda^{2}(\nu+\lambda)(\nu+ & \lambda+2 \mu) y \\
& +\lambda^{2} \mu^{2}(\lambda+\mu)(\lambda+\mu+2 \nu) z=0 ;
\end{aligned}
$$

and the lines joining these points of intersection to the vertices are

$$
x / \lambda^{2}+y / \mu^{2}=0, y / \mu^{2}+z / v^{2}=0, z / v^{2}+x / \lambda^{2}=0 .
$$

In Fig. 12, the outer (dotted) triangle is the original triangle of reference, and the inner triangle is the new triangle of reference. If the given point lie in the space $\mathrm{A}$, or in a space $\mathrm{E}$, the tangents at the double point are imaginary, and the point is a conjugate point; if the given point lie in any of the other spaces, the tangents are real.

The form of the curve depends upon the position of the given point, as indicated in Fig. 12. When the given point is in any of 
the spaces marked with the same letter, the form of the curve is essentially the same. When the point lies on any of the lines of the figure, or on the straight line at infinity, the cubic breaks up into that straight line and a conic section; when the point coincides with any of the points of the figure, or with one of the three points of intersection of the lines of the figure with the straight line at infinity, the cubic breaks up into three straight lines. When the given point coincides with one of the vertices of the outer triangle, the three straight lines are the three sides of that triangle; when it coincides with one of the vertices of the inner triangle, or with one of the three points at infinity, the three straight lines are the three lines of the figure that pass through the point under consideration.

When the given point is at infinity, since an asymptote of the circumscribed conic passes through the given point, the conic itself passes through this point; hence the conic itself passes through four given points, and the locus of its centre is a nine-point conic; unless the circumscribed conic be a parabola, in which case the locus of the centre is obviously the straight line at infinity.

The other two degenerate cases are connected with the facts that the isotomic conjugate of a side of a triangle is any straight line through the opposite vertex, and that the straight line joining the middle points of two sides of a triangle is its own isotomic conjugate.

I have traced the curve for a position of the given point in each of the spaces $A, B, C, D, E$; and also in three of the degenerate cases, in order the better to show how one form of the curve passes into another. In all the figures the exterior (dotted) triangle is the triangle originally given, the inner triangle is the triangle of reference; and $P$ is the given point, and is the double point on the cubic.

In Fig. 13, $\mathrm{P}$ is the centroid $(1,1,1)$; the asymptotes are all intlexional, and the cubic touches each side of the exterior triangle at its middle point.

Fig. 14, in which $P$ is the point $(0,4,5)$ illustrates the more general case, for the space $A$, in which no asymptote is inflexional.

In Fig. 15, the degenerate case is represented when $\mathbf{P}$ is a point on a line between the space $A$ and a space $B$. The curve consists of a hyperbola and the line : 
In Fig. 16, the point $P,(2,2,-1)$, is in a space $B$; as this point has been taken on one of the medians, one of the asymptotes is inflexional.

Fig. 17 represents the degenerate case when $P$ lies on a line between a space $B$ and a space $C$.

In Fig. 18, the point $P,(2,4,-3)$, is in a space $C$.

Fig. 19 represents the degenerate case for the point $P$ on a line between a space $C$ and a space $D$.

In Fig. 20, the point $P,(8,8,-9)$, is in the space D. [In this figure, the two separate infinite branches have been drawn considerably nearer to the triangle than they should be.]

In Fig. 21 , the point $\mathbf{P}(-1,-1,4)$ is in the space $\mathrm{E}$.

It has not been thought necessary to draw the figures for the other two degenerate cases, namely, when the point $P$ is on a line between a space $C$ and a space $E$, and when the point $P$ is at infinity. 